\title{
La presse francophone dans l'Empire ottoman et la Turquie de Mustafa Kemal
}

French speaking Press in Ottoman and Mustafa Kemal's Turkey

Osmanlı İmparatorluğu ve Mustafa Kemal Türkiye'de Fransız basını

\section{Gérard Groc}

\section{OpenEdition}

\section{Journals}

Édition électronique

URL : https://journals.openedition.org/ceb/16007

DOI : $10.4000 /$ ceb. 16007

ISSN : 2261-4184

Éditeur

INALCO

Édition imprimée

ISBN : 9782858313693

ISSN : 0290-7402

\section{Référence électronique}

Gérard Groc, «La presse francophone dans l'Empire ottoman et la Turquie de Mustafa Kemal »,

Cahiers balkaniques [En ligne], 47 | 2020, mis en ligne le 21 août 2020, consulté le 06 juillet 2021. URL http://journals.openedition.org/ceb/16007 ; DOI : https://doi.org/10.4000/ceb.16007

\section{(c) (1) (5)}

Cahiers balkaniques est mis à disposition selon les termes de la Licence Creative Commons Attribution - Pas d'Utilisation Commerciale 4.0 International. 


\title{
La presse francophone dans l'Empire ottoman et la Turquie de Mustafa Kemal
}

\author{
French speaking Press in Ottoman \\ and Mustafa Kemal's Turkey \\ Osmanlı Imparatorluğu ve Mustafa \\ Kemal Türkiye'de Fransiz basin
}

Gérard Groc

Chercheur associé à l'IRENAM

(Institut de recherches et d'études_sur le monde arabe et musulman)

Les débuts de la République turque, proclamée en octobre 1923, sont, pour la presse en français qui existe en Turquie depuis fort longtemps, un renversement des conditions d'exercice. Ils marquent le passage d'un ancien régime - où cette presse connaissait depuis plus d'un siècle un succès pérenne et avait acquis ses habitudes - à un nouveau régime. Processus révolutionnaire, transformateur et iconoclaste puisqu'il procède d'une guerre d'indépendance où une rébellion nationaliste reconquiert pas à pas (mai 1919-août 1922) un territoire dont la Première Guerre mondiale, une mésalliance avec la Prusse et la défaite avaient réduit la consistance. Soulignons de suite que le territoire reconquis n'est plus celui de l'Empire ottoman avec ses dépendances balkaniques, arabes et musulmanes, mais un territoire restreint à l'Anatolie et une partie de la Thrace, pressenti dès février 1920 (Pacte national) comme le cœur d'une nouvelle souveraineté, celui qu'il convient de rebâtir pour effacer les humiliations et les spoliations de la défaite consignées par le traité de Sèvres (août 1920).

Convaincue de la nécessité d'une communication, cette rébellion utilise immédiatement la presse, d'abord en interne, pour une mobilisation « démocratique », large et représentative de toutes les provinces contre un 
pouvoir resté dans une capitale sous occupation étrangère (Constantinople), mais aussi en externe car son chef, Mustapha Kemal, sait d'emblée qu'une reconquête ne sera valable qu'entérinée par les vainqueurs du conflit mondial. Le premier véritable organe de presse est mis en place en janvier 1920 sous le titre injonctif de Souveraineté nationale (Hakimiyeti Milliye). Un tel recentrage des priorités vers l'affirmation d'une primauté nationale et l'exigence d'une reconnaissance d'indépendance relativise forcément l'utilité d'une presse de langue étrangère, éditée de surcroît dans la langue d'un des vainqueurs. Pourtant, l'usage perdure et, une fois la paix retrouvée et la république proclamée, une telle presse continue d'exister y compris dans une grande diversité des styles; mais son utilisation doit se soumettre bientôt aux conditions édictées par une volonté politique de plus en plus centralisatrice, au point d'en devenir peu à peu un instrument de propagande. Là où on connaissait un large éventail d'expressions, reflétant autant une multiplicité d'interlocuteurs extérieurs mais présents sous une forme ou sous une autre dans l'Empire, qu'une pléiade de publicistes internes désireux, chacun pour ses raisons propres, d'utiliser la langue française pour s'exprimer, on assiste vers le tournant des années 1930 à la captation croissante de cet usage au profit du seul appareil gouvernemental. Ce dernier est soucieux désormais de faire entendre vers l'extérieur non seulement les progrès qu'il accomplit vers la « civilisation » mais, plus explicitement, la position qu'il compte adopter face aux déploiements expansionnistes de certains régimes européens, comme l'Italie, dont il craint les élans invasifs dans l'aire méditerranéenne.

On voit donc qu'une presse en français dans la Turquie des années 1920 et 1930 combine l'articulation de plusieurs paramètres, au rang desquels d'abord l'impératif d'une indépendance nationale, puis le rôle que doit y tenir une presse nationale, enfin une langue étrangère et l'utilisation qu'il convient d'en faire. Et vers quel public?

En partant de l'intérêt que présente une presse « allophone » dans l'évaluation d'une trajectoire national(ist)e - au-delà d'une seule production d'exil, de migration ou encore de communautés marginalisées, repliées sur des intérêts particularistes -, il est intéressant de situer les modalités de son insertion dans le schéma dominant, de mesurer sa contribution à cette trajectoire : dans quelle mesure l'enrichit-elle de ses nouveautés, de ses questionnements, quelle ouverture lui procure-t-elle, quels défis lui pose-t-elle ? Et partant de là, il est intéressant aussi de retracer les débats collectifs ou individuels dans lesquels s'inscrivent les publicistes qui en deviennent ipso facto des protagonistes, représentant par choix linguistique délibéré sinon des points de vue du moins des perspectives différentes et parfois concurrentes. Une presse allophone représente a priori un « extérieur » qui regarde, évalue, critique une évolution en cours, s'y attache aussi d'une 
certaine manière puisqu'il choisit d'inscrire son activité dans son cadre, mais s'en dissocie en même temps, adoptant le regard d'un « autre » sur la base de critères politiques, culturels et prioritairement linguistiques divergents. Ce qu'offre en plus la presse en français de Turquie (cette dernière prise au sens large d'Empire ottoman et de période républicaine), c'est qu'elle est doublement allophone, une sorte d'allophonie « au carré » puisqu'elle abrite en son sein, outre des interlocuteurs français représentant des intérêts français - ce que l'on pourrait nommer une « presse française » - d'autres locuteurs qui ne sont pas eux-mêmes francophones, qui sont en réalité de cultures multiples, mais qui choisissent néanmoins cette langue pour se positionner dans l'espace public ottoman ou turc et publier ce qu'ils ont à exprimer. Ce peut être des Turcs, et donc turcophones, qui choisissent la langue française pour produire un discours différent de celui de leurs compatriotes ; ce peut être aussi des locuteurs non turcophones, ottomans ou étrangers, mais présents dans le lieu, qui vont exprimer, dans une autre langue que la leur et pour un public qui n'est pas non plus un locuteur direct de la langue française, ce qu' ils veulent porter à la connaissance du public. L'un et l'autre cas ne sont pas exceptionnels, mais c'est leur réunion dans un même contexte qui en fait l'intérêt, leur juxtaposition dans le même temps et espace et dans la même langue, créant, au-delà de leur différence culturelle, non seulement une communauté linguistique spécifique mais une approche et une contribution à la fois différenciée et simultanée, à l'évidence complémentaire, envers le même phénomène. Quels que soient le point de départ et les modalités de cette expression, elle procède, dans toute sa dimension, de l'utilisation d'un medium culturel moderne, soucieux de se positionner dans l'évolution collective, impériale ou républicaine, et néanmoins « nationale ». L'emploi de la langue française dans l'activité de presse en Turquie est en effet un phénomène considérable, novateur et structurant.

\section{L’emploi de la langue française dans la presse ottomane}

Historiquement, c'est en français que naît le premier périodique de Turquie, à Constantinople, les Bulletins de Nouvelles du représentant de la Convention nationale, dès la fin de 1793, pour une activité qui se poursuit jusqu'en juin 1797. L'intérêt de ce premier épisode de presse est qu'il revêt une grande variabilité de formes (quatre titres), se fait en français, mais aussi en traduction en plusieurs langues, connaît des régimes divers (clandestin, toléré puis officiellement reconnu) avec des statuts différents (presse de propagande puis presse diplomatique, enfin, organe privé au service d'intérêts dits commerciaux), autour d'une intention prioritaire de sensibilisation aux progrès de la Révolution française. Cet épisode reste sans lendemains immédiats. 
Historiquement, c'est encore en français que s'institue, un peu plus tard à Smyrne, en 1821, aux mains de citoyens français privés, une activité journalistique régulière, avec rubriques, avis, informations commerciales, culturelles et prises de positions politiques. Même si les titres se succèdent (Smyrnéen, Spectateur Oriental puis Courrier de Smyrne), l'activité, elle, ne s'interrompt plus. Et c'est à partir de cette innovation provinciale qu'aura lieu, au début de novembre 1831, à Constantinople, la création d'un organe officiel impérial, le Takvim-i Vekayi, en turc, voulu par le Sultan, immédiatement doublé d'une version francophone intitulée Le Moniteur ottoman, et confiée au rédacteur du Courrier de Smyrne, débauché pour l'occasion. Là où la version turque se borne à la mention des actes officiels, la version française s'ouvre à la discussion et à la représentation argumentée des intérêts de l'Empire, contrant même parfois les intérêts français. Jusqu'en 1836, date de la mort de son rédacteur français, Alexandre Blacque, elle est aussi une importante source d'informations pour les Européens sur la situation politique au Moyen Orient ${ }^{1}$. Même en version française, ce journal reste cependant aux yeux de ses confrères européens de l'époque, un journal officiel du Sultan.

Historiquement, c'est à nouveau en français, et encore une fois à partir d'un transfert cumulé de deux journaux smyrniotes, que s'établit dans la capitale en 1843 et sur plus de 20 ans, une véritable activité journalistique francophone, qui se veut informative et d'opinion, avec le Journal de Constantinople... d'abord défini comme celui ...des Intérêts orientaux, devenu ensuite ...Écho de l'Orient. C'est une presse cette fois privée, française par facture journalistique (les rédacteurs en chef viennent de France, elle est parfois financée par l'ambassade de France), mais directement en faveur de la Porte, ottomane par engagement et par devoir, chargée de promouvoir l'introduction formelle de l'Empire dans la sphère politique occidentale. Les années 1850 voient la création de cinq autres journaux francophones alors que n'existent, comme journaux turcs, que le journal officiel en édition turque et arménienne, et un autre journal, fondé en 1846 par un... Anglais. La francophonie détient donc indéniablement, et jusqu'à la fin des années 1860, un quasi monopole d'information et d'expression, car le français vaut comme langue de communication dans une adéquation choisie entre la volonté politique ottomane et l'option de la langue française. En 1851, le Journal de Constantinople a même l'audace de réclamer l'abolition des Capitulations et propose à la Porte d'édicter un droit commun en matière de commerce et d'industrie, à la grande contrariété des puissances occidentales qui ignorent superbement cette demande. En retour, le journal s'efforce de renseigner sur les innovations politiques 
occidentales et surtout françaises et, de fait, beaucoup de réformes ottomanes $s$ 'inspirent alors de dispositifs français voire se rédigent en français ${ }^{2}$.

En réalité, la francophonie est à cette époque beaucoup plus large qu'un fait journalistique. Durant ce qu'on appelle les Tanzimat (《Les réformes »), grosso modo de 1839 à 1876, le français est une langue de la diplomatie pour la Turquie mais aussi pour l'Autriche et la Russie. La nouvelle élite bureaucratique ottomane fait son apprentissage dans le Cabinet des Traductions (Tercüme Odasi) où tout se rédige en français. Les trois grands personnages de cette période jusqu'en 1870, chacun plusieurs fois ministre ou grand vizir - Rechid, Fuad et Ali Pachas - sont d'excellents francophones et maints documents ottomans sont par eux initialement rédigés en français ${ }^{3}$. Le français devient aussi une langue d'éducation dont l'administration se soucie beaucoup ; d'ailleurs, le lycée Galatasaray, francophone, créé en 1868 et inauguré par Victor Duruy, deviendra bientôt la pépinière des intellectuels et des commis de l'État. Pour l'Empire, la langue française n'est donc plus seulement une langue d'intérêts particuliers mais bien plutôt celle qui dépasse les particularismes, va plus avant dans la civilisation étrangère et moderne et expose les progrès réalisés.

Dans cette évolution, les années 1850 introduisent une nouvelle configuration plus contraignante. La guerre de Crimée installe les étrangers plus directement dans l'Empire. À son terme, la Turquie se retrouve sommée en 1856 (traité de Paris) d'accorder plus de droits à des groupes minoritaires qui formulent déjà des revendications nationalistes voire sécessionnistes (l'exemple grec est dans toutes les têtes). En retour, une opinion publique ottomane s'ébauche qui commence à dénoncer les surenchères occidentales visant au démembrement de l'Empire. Les affrontements du Liban, de Serbie et de Crète émaillent déjà l'actualité. En guise de contestation, une question, celle d'une identité ottomane, taraude la première génération de journaux véritablement turcs qui publient, dans la décennie 1860, les premiers périodiques en turc et non plus en français, avant de succomber aux fourches de la censure (loi sur la presse en 1865) et de chercher refuge, qui en France, qui à Londres. Bientôt sonnera aussi la défaite de la France face à la Prusse, et le recul de ses performances institutionnelles, d'autant qu'elle même se tourne de plus en plus vers des formes républicaines. Autrement dit, le message d'une France officielle se ternit quelque peu, au milieu des années 1870.

Évolution lente, mais irrémédiable, où une presse foncièrement française change alors de dimension pour se fondre dans une évolution d'ensemble multilingue où elle ne joue plus un rôle prioritaire. L'utilisation du français

2. Groc, 1992, p. 15-28.

3. Davison, 1990, p. 125-141. 
n'y est plus un phénomène global ni l'effet d'une volonté politique ottomane, mais devient peu à peu le choix délibéré de particuliers qui, à côté d'une presse désormais possible en d'autres langues, choisissent pourtant de s'exprimer en français. D'exclusif, la francophonie devient un phénomène sélectif, et ceci dans un contexte spécifiquement turc. À partir de là, l'étude de la presse francophone devient une série de cas d'espèces qui nous commande de trouver pourquoi, dans un tel contexte, telle personne choisit à telle époque d'éditer un organe en français. Critères politiques ? Simples critères culturels ? Volonté d'ascension sociale ? Phénomène de mode ? On est donc obligé de parler d'une espèce de banalisation $\mathrm{du}$ fait francophone au sein même de l'évolution journalistique où l'usage de la langue française relève d'un appoint culturel et civilisationnel.

La tendance s'accentue sous le long règne d'Abdülhamid II (1876-1909), où le pouvoir contrôle de plus en plus l'activité de presse dont il veut brider toute portée politique. Il est vrai qu'à partir des années 1880 s'ébauche une contestation étudiante que son envoi en exil transforme en une opposition de plus en plus structurée, du nom de jeune-turque, dont l'écho deviendra vite international. Dans ce contexte d'une presse muselée, la francophonie devient plutôt un véhicule culturel pour une foule de revues à périodicité plus espacée, entreprises individuelles à capacité financière plus ou moins réduite, à valeur plus spécialisée et à durée de vie plus ou moins courte. Le Stamboul, journal de langue française qui vaut, avec le recul, comme un monument sinon de l'influence du moins de la présence française, est un bon exemple de cette diversité des genres ${ }^{4}$. En réalité, ce journal, paru en août 1875, est au début un journal édité par un Irlandais, John Laffan Hanly, succédant au Progrès d'Orient du même éditeur, valant pour représentant des intérêts anglais en Turquie. Ce n'est qu'en 1901 qu'il est rédigé par un Français, R. Delbeuf ; en 1908, il devient « journal français paraissant en turc » et en 1910, il est définitivement un « organe français ». De fait, sur les quelque 40 publications francophones de l'ère hamidienne (1876-1909), la plupart sont des bulletins économiques de chambres de commerce, des revues médicales, d'annonces, ou encore des revues littéraires mondaines ou artistiques où Victor Hugo côtoie les échos de la mode parisienne. 1880 apporte une innovation, L'Osmanli, organe commandité par la Porte, s'adjoint une édition en langue française à laquelle de grandes signatures participent. D'autres initiatives plus modestes voient le jour à partir du Palais, souvent sous forme de revues plus ou moins richement illustrées. La bride est telle néanmoins qu'en 1905, à côté des six journaux en français, des douze quotidiens en turc, des neuf en grec, etc., le public cosmopolite de Constantinople préfère puiser directement dans les 145 titres

4. Alemdar, 1975. 
français, 34 titres allemands, 14 titres autrichiens, 4 titres belges, etc., quotidiens, hebdomadaires ou autres périodiques reçus régulièrement de l'étranger à plusieurs milliers d'exemplaires 5 .

À noter que le souffle de la révolution jeune-turque de 1908 déclenche une avalanche de publications qui voient le jour ou tout au moins reçoivent une autorisation de publication, parmi lesquelles 94 sont francophones, parfois aussi en formule trilingue, voire quadrilingue. Il est vrai que, pour quelque temps, l'atmosphère est encore à la liberté et à la réconciliation de toutes les dimensions de cet empire.

\section{Après la Première Guerre mondiale}

Aux lendemains chaotiques de la Première Guerre mondiale, au moment où les vainqueurs fixent les conditions de survie d'un empire défait et où s'ébauchent les germes d'une résistance nationale qui en liquidera bientôt les fondements, la presse francophone continue d'exister et de présenter un visage très diversifié, mais elle est en pleine recomposition. C'est une combinaison d'organes aux intérêts multiples qui appelle une approche elle-même différenciée. On peut y dénombrer trois périodes.

\section{8-octobre 1923}

La première période étendue de la fin de la guerre à octobre 1923, date de la proclamation de la république, est une période confuse où les opinions ont du mal à se départager. On reste sur l'image et un héritage d'Empire, tout en sachant que, par-delà la défaite, les choses ne seront bientôt plus les mêmes. D'abord, la Turquie ne sait pas à quelle sauce elle sera accommodée par les vainqueurs. Les pourparlers pour les questions d'Orient passent après la réorganisation des champs de guerre européens, et ce n'est qu'après la signature du traité de Versailles en juin 1919 (entré en vigueur en janvier 1920), puis le traitement de l'Autriche, Bulgarie et Hongrie, que les vainqueurs s'intéressent entre autres au sort de la Turquie, soit au tournant de l'année 1920. Entretemps, Constantinople est occupée par les troupes anglo-franco-italiennes; les troupes grecques ont débarqué à Smyrne en mai 1919 pour faire en Anatolie le travail de police que les vainqueurs ne veulent plus faire directement et, en riposte immédiate, une révolte nationaliste turque se développe dont le succès et l'organisation iront croissant. Les interrogations qui font une grande part de l'actualité sont nombreuses. En quelle proportion l'Empire sera-t-il démembré ? La Turquie sera-t-elle placée sous un mandat américain, dans

5. Revue Commerciale du Levant, $\mathrm{n}^{\circ}$ 201, août 1905. 
la suite de la déclaration Wilson, ce dont on parle en octobre 1918 ? On pressent assez vite, même sans connaître forcément l'existence du pacte Sykes-Picot, dévoilé en février 1920, que certaines provinces arabes seront détachées de l'Empire. En ira-t-il de même de provinces chrétiennes, au moment où des pays caucasiens, Arménie, Azerbaïdjan et Géorgie proclament leur indépendance (16 janvier 1920, ouverture de la SDN) ? Qui se fera le protecteur des «petites nations » ? Les populations chrétiennes et juives de l'Empire conserveront-elles leur statut de minoritaires ? En janvier et février 1920, les journaux parlent de nouveaux massacres de 20000 Arméniens à Marache, par les Nationalistes. On connaît aussi l'existence de la déclaration Balfour (novembre 1917) qui, vers la fin 1919, divise les populations juives de l'Empire et pose la question de leur départ vers la Palestine et plus globalement du sionisme (La Nation de Nissim Rousso, premier numéro le 24 octobre 1919). En février 1920, la Conférence de Paris décide pourtant que Constantinople restera sous souveraineté turque, ce qui n'empêche pas l'Angleterre d'y resserrer son contrôle militaire le 16 mars 1920.

L'opinion publique ottomano-turque, bigarrée, se fragmente sur ces incertitudes que chacun interprète à sa manière, et les communautés levantines et minoritaires, dont l'usage du français dans les publications est courant ( $L e$ Bosphore de Michel Paillares, quotidien qui apparaît le 22 octobre 1918, Le Journal d'Orient d'Albert Carasso, quotidien paru durant l'été 1918), cherchent avant tout à se concilier les puissances occidentales, tout en sachant qu'elles restent parties intégrantes d'un Empire en faillite. La rébellion nationaliste, pour sa part, désire, par l'utilisation de congrès « nationaux », faire reconnaître sa lutte et s'appuie, elle aussi, sur le point 14 de la déclaration Wilson, celle du droit des peuples à disposer d'eux-mêmes. Cette rébellion, qui conquiert peu à peu l'Anatolie lointaine, fragilise encore plus les observateurs restés à Constantinople, au chevet de l'Empire. C'est à la mi-mai 1920 que sont connues les conditions de paix imposées par les vainqueurs qui seront ensuite consignées dans le traité de Sèvres (août 1920). « Très dures » commente Le Journal d'Orient du 15 mai 1920 : Smyrne placée sous administration grecque, Syrie et Mésopotamie gérées en mandat, une Arménie et un Kurdistan libre. «Tout crie qu'on ne veut pas la mort de la Turquie! » veut-on se rassurer. Et dès l'été 1920, les premiers revers de l'armée grecque dans ses tentatives d'occuper le centre du pays (l'armée nationaliste reçoit le soutien de la nouvelle URSS en contrepartie de la délimitation d'une frontière commune dans le Caucase) font vaciller encore plus fort les quelques certitudes dont certains publicistes osent se prévaloir.

Lorsqu'on parle alors de presse francophone, c'est un mélange de presse informative et spécialisée, commerciale et professionnelle (Revue commerciale d'Orient $n^{\circ} 25, L^{\prime}$ information d'Orient $\mathrm{n}^{\circ} 16$ signalé en février 1920), économique 
(L'Économiste d'Orient paru le 12 février 1920), et en grande part, d'une presse minoritaire encore installée dans le reflet d'un cosmopolitisme hérité de l'Empire ; tout autant de publications qui, au sortir de la guerre et d'une longue présence prussienne, tentent conjointement de revivre, de rebâtir des activités et de renouer avec la paix. Mais le statut du français varie aussi avec les louvoiements de la France elle-même qui, dans cette phase de transformation, tient à ses interlocuteurs plusieurs discours, assume plusieurs rôles entre lesquels il n'est pas facile de faire un tri immédiatement rationnel. D'une part, elle maintient son plus beau fleuron, $L e$ Stamboul, lequel propage encore la vision d'une continuité colonialiste et milite pour le maintien des intérêts français. Pour lui, la France est d'abord un vainqueur qui œuvre, avec ses alliés européens, à la liquidation de l'Empire ottoman, impose à ce qu'il en reste les rigueurs d'un démembrement que l'on discute tout au long de l'année 1920 : la Turquie y est réduite à un petit territoire au centre anatolien. La France, puissance impérialiste, anciennement bénéficiaire des Capitulations auxquelles les nationalistes opposent l'affirmation d'une souveraineté nationale, préserve donc ses intérêts dans le Levant. Sur un autre plan, elle est aussi le pays d'une Révolution française largement inspiratrice pour les leaders kémalistes, inventeur en 1793 de mobilisations patriotiques et de victoires obtenues, face aux coalitions existantes, par désir de liberté, capable de secouer le joug de pouvoirs anciens et délégitimés par leurs échecs. C'est le pays d'une expérimentation républicaine, pourvu de dispositifs innovants tels que l'instruction publique obligatoire et gratuite ou encore la laïcité, dont le kémalisme va grandement s'inspirer. C'est donc aussi un partenaire de la modernité. Enfin, en 1920, la France est également une force militaire d'occupation dont les troupes, installées dans le Sud anatolien, tiennent des territoires où elle se fait précisément le champion d'une restauration arménienne en réponse au génocide de 1915. C'est d'ailleurs contre elle que porteront les premiers faits d'armes de la résistance nationale turque (Sütlü imam, Marache « l'héroïque »), ce qui ne l'empêche pas de devenir très rapidement un interlocuteur de ces rebelles qu'elle combat et de rechercher leur alliance, car son autre et alors véritable intérêt se situe en Syrie, hors du territoire national turc, où elle veut conforter sa situation de puissance mandataire face à l'Angleterre. Son souci devient vite, au-delà de la Cilicie, la fixation de frontières claires, ce qui la pousse à renverser ses options d'envahisseur pour devenir le premier signataire d'un accord (octobre 1920) avec le nouveau régime républicain. Cette amitié très tôt manifestée ${ }^{6}$ (Briand, colonel Mougin) va permettre aux rebelles de casser le front des vainqueurs et de programmer les étapes d'une reconquête territoriale qui se fera d'est en ouest. Dès 1920, les envoyés français, plus ou moins officiels, se

6. Le Temps, en octobre 1919, publie une interview de Mustafa Kemal. 
succèdent, directement en contact avec la rébellion kémaliste et les relations qu'ils en feront en France, dithyrambiques, provoqueront rapidement un capital de sympathie, porté par des hommes éminents tels que Édouard Herriot, lui-même devenu un familier de Mustafa Kemal'

On l'a dit, cette première période se solde cumulativement par la victoire nationaliste à l'été 1922, puis par sa reconnaissance politique par le nouveau traité de Lausanne, négocié dès la fin 1922 et signé en août 1923, qui abolit les amputations territoriales de 1920, puis par la proclamation d'une république en octobre suivant.

\section{Les débuts de la République turque, 1923-1930}

Dès lors, les choses changent, car la Turquie entre dans une période de construction républicaine qui redistribue les circuits du pouvoir, entraîne la confection de nouvelles institutions autour d'une souveraineté populaire incarnée par une assemblée nationale. S'y réalise assez vite l'abolition de l'édifice impérial (le sultanat en octobre 1922, le califat en avril 1924), à travers quelques nouvelles lois fondamentales sur l'organisation de l'exécutif, de la justice, de l'éducation nationale, et la rédaction d'une nouvelle constitution, prête en avril 1924.

En ce qui concerne la presse, la nouvelle capitale Ankara entend faire rapidement reconnaître sa centralité nouvelle, ce qui l'amène à épingler puis forcer les journaux de Constantinople, les plus nombreux, les plus connus et les plus talentueux, à se mettre au service du nouveau régime et à abandonner leur ancienne quête de diversité voire de célébrité. La manœuvre dure de 1923 à 1925 , conciliante au début. Puis, au déclenchement de la révolte kurde du Cheikh Saïd (février 1925), l'injonction politique se fait autoritaire, des journaux sont fermés, des directeurs de publications condamnés et bientôt relâchés. Mustafa Kemal précise alors, dans son discours d'ouverture de la session parlementaire : « Il ne peut y avoir de place pour les journaux qui troublent la tranquillité de la Nation, qui sèment l'erreur et se livrent à de mauvaises critiques $\gg$. La presse est quasiment bâillonnée.

Sur un total de 251 publications en 1925, 98 paraissent à Constantinople contre 12 respectivement à Ankara (dont 2 quotidiens) et à Smyrne, le reste se répartissant sur l'ensemble du nouveau territoire. Pour ce qui concerne une presse en langue non turque, reflet d'une diversité ethnique, confessionnelle et sociale héritée de l'Empire, à deux exceptions près (Smyrne et Andrinople), la totalité des journaux en langue non turque (34) paraît à Constantinople. 12 (?) sont en français dont 5 quotidiens (Stamboul, République, La Gazette [Gattegno/Primi], 
La Volonté, le Journal d'Orient), 4 en grec, 11 en arménien dont étonnamment 8 quotidiens, 4 en judéo-espagnol, 2 en russe et un en italien. On constate la disparition totale d'une presse anglaise et allemande. Hors Istanbul disparaît aussi toute presse grecque et arménienne ${ }^{8}$.

C'est une époque où la situation sociale se tend du fait qu'existent, d'une part, les garanties prévues pour les minoritaires et reconduites dans le nouveau traité de Lausanne (articles 37 à 42) concernant la conservation de l'usage de la langue, l'exercice de la confession, la conservation des lieux de culte et l'octroi d'un statut personnel exceptionnel et, d'autre part, que le régime nationaliste se fait de plus en plus insistant dans sa volonté d'instaurer un modèle républicain de droit commun, égalitaire, culturellement turc (forcément turcophone), fondamentalement rétif à toute mention de particularisme. Cette tension place les minoritaires dans un dilemme profond animé par la peur de s'isoler de l'expérience naissante; elle est assez forte pour les pousser, dès 1924, à renoncer successivement à un statut personnel particulier et à vouloir se couler dans celui d'une citoyenneté nationale. Pour compléter le tableau, il faut ajouter le fait que les populations grecques de Turquie sont d'autant plus réduites qu'il est procédé en 1923 à un transfert de population avec la Grèce, qui voit un million de Grecs quitter la Turquie et principalement la Cappadoce, contre 400000 musulmans qui font le chemin inverse. Quant à la population arménienne, à part une infime part regroupée à Constantinople qui tient à marquer sa présence par une activité de publication prolifique, la presque totalité a succombé au génocide de 1915. On le voit, la tendance n'est plus à la survie d'une pluralité culturelle et encore moins politique. Seule continue à paraître une rare presse juive (Courrier de Turquie 1919, L'écho d'Andrinople 1921, Hamenora 1923) qui est d'autant mieux tolérée qu'elle ne constitue aucune menace territoriale sur la nouvelle Turquie et que quelques-uns de ses ténors ont embrassé l'enthousiasme nationaliste?.

Pour ce qui est de l'usage du français, sur l'heure, peu de journaux indépendants voient le jour (La Turquie, revue mensuelle de Salon et publicité 1924, Opéra Ciné 1924, $P S^{\prime} T$, journal humoristique 1924). Par contre, c'est l'avènement des éditions en français de journaux turcs, rentrés peu à peu dans le rang, qui marque alors un certain rebond d'une presse francophone. En 1924 paraissent L'Akcham puis Le Tanine, francisant à peine leurs titres, suivis de La République (Cumburiyet en turc), puis du Milliyet qui deviendra La Turquie en 1933. Leur utilité réside désormais dans la transcription de la rhétorique gouvernementale d'abord vers ces

\section{Deny, 1925 et 1933.}

9. Son plus beau fleuron est Le Journal d'Orient déjà cité plus haut, lancé à la fin de 1919 qui traversera les époques jusqu'en 1971. 


\section{CAHIERS BALKANIQUES}

populations minoritaires qui ne parlent souvent pas encore le turc (en 1928 sera autoritairement lancée la campagne de turcisation de la vie publique sous le mot d'ordre Vatandaş, türkçe konus [ « Citoyen, parle turc ! »]), puis vers un auditoire étranger présent sur le territoire et en dehors, que le gouvernement d'Ankara veut informer de ses prérogatives nationales ${ }^{10}$. Quelques parutions scientifiques ou économiques, comme L'Économiste d'Orient 1920 de R. S. Atabinen, menées par des intellectuels ralliés, ont pour tâche quant à elles d'afficher l'itinéraire positiviste suivi par la nouvelle république, que véhiculent aussi les nombreuses réformes entreprises entre 1925 et 1929.

La position de la France reste encore ambivalente. Le Stamboul continue de paraître prêtant assez peu de crédit aux réformes en cours. Toutefois, les diplomates français en poste en Turquie deviennent plus attentifs aux évolutions qui marquent le pays [en 1924, Édouard Herriot, soutenu par le Cartel des gauches, est alors devenu chef du gouvernement] et décident en août 1925 la confection d'un Bulletin de la presse turque, qui suit 26 journaux turcs de plusieurs origines et sensibilités, en traduit les principaux extraits pour mieux appréhender les pulsations de la nouvelle nation et en transmettre hebdomadairement la teneur aux autorités de Paris. Le nouveau régime n'en reste pas moins très ombrageux et n'hésite pas à animer contre « son ami français » des controverses relatives soit aux questions de la frontière syrienne soit au remboursement des coupons de la dette ottomane ou encore à des conflits de compétence comme en soulève l'affaire Lotus-Bozkurt à propos d'une collision maritime pour laquelle Paris dénie à la Turquie le droit de la juger ${ }^{11}$.

\section{Après 1930, une réorganisation autoritaire}

Tout change après 1929/1930, ce qui marque la troisième période indiquée au début. Ankara est enfin libérée des contraintes des clauses temporaires du traité de Lausanne. Tirant aussi les conséquences de la crise de 1929 et voulant éliminer toute vulnérabilité face aux intrusions extérieures, elle entame une période de réorganisation politique autour de l'idée d'un gouvernement autoritaire $^{12}$, concentrant tous les circuits de la décision politique, guidé par un parti unique débarrassé de toute opposition et de tout obstacle à sa marche en avant, constructeur d'une autarcie économique ; l'État non seulement entoure son activité économique des protections nécessaires concernant ses activités

10. GEORGEON, 1985, p. 27-40.

11. Groc, 1986, p. 479-498.

12. Boratav, 1981 , p. 146-165. 
commerciales, bancaires, financières, budgétaires, etc., mais il devient lui-même un entrepreneur de premier plan, agençant autour de ses entreprises les modalités de la nouvelle économie nationale. Et, à partir de là, sa position évolue selon deux objectifs au service desquels une presse francophone, d'une nouvelle mouture, va s'organiser. D'abord, il revendique hautement la reconnaissance de son indépendance et veut montrer que l'édification d'un nouveau pays, d'une nouvelle société est en cours. L'année 1933, dixième anniversaire de la République, donne lieu à des célébrations où sont présentées les innovations qui encadrent désormais le quotidien du pays. Ensuite, il veut inscrire son pays au rang des pays dont la souveraineté doit être reconnue, à la recherche d'une parité, et ce, au spectacle d'une évolution belliciste qu'il constate en Italie et en Allemagne et dont il craint les appétits conquérants (1929, déclaration d'une expansion coloniale de l'Italie vers la Libye et l'Abyssinie ; 1933, pression allemande sur les détroits pour gagner la Turquie à un pacte germano-italien). Sujette aux tensions qui en résulterait, la Turquie envisage déjà de rester neutre en cas de conflit européen (1936, tension avec l'Angleterre à propos de Mossoul et refus russe de lui reconnaître la souveraineté dans les détroits octroyée en 1935 par la convention de Montreux) de faire accepter par tous cette neutralité, pour ne pas subir à nouveau l'issue désastreuse que lui a réservée la Première Guerre mondiale. Dans ce contexte, une presse francophone continue bien d'exister, mais elle s'enrichit, en cela qu'à côté des éditions françaises des journaux turcs qui reproduisent encore les slogans du régime, apparaissent des revues nouvelles, périodiques promotionnels, richement illustrés où la nouvelle république décline ses créations, ses nouveautés, ses chantiers et constructions nouvelles et veut donner à entendre qu'elle est en phase de se doter des équipements architecturaux, scientifiques, institutionnels, administratifs qui font la modernité du pays et le promeuvent au rang des nations modernes. C'est l'époque de la création d'influents instituts scientifiques comme l'Institut d'histoire turque créé en 1931 ou l'Institut de langue turque qui suit en 1934, qui sous-tendent ensuite la série des Congrès d'Histoire (1934, 1937) qui vont théoriser les socles indéfectibles d'une identité turque et l'idée d'une antériorité de la civilisation turque, mêlant découvertes archéologiques, réflexions sur l'histoire et promotion de la littérature turque. Alors, autour de cette idée, on va voir peu à peu fleurir, en français, des publications de belle facture, illustrées de nombreuses photos, directement liées au régime et à ses services de propagande, au ton hagiographique, et qui portent haut les réalisations de l'édification d'une nation nouvelle et dont les titres signalent déjà par eux-mêmes l'intention de leurs éditeurs (La Turquie rénovée 1930, Les Annales de Turquie 1931, La Turquie 


\section{CAHIERS BALKANIQUES}

28

La presse allophone dans les Balkans

Kémaliste $1934^{13}$, L'Illustration de Turquie 1934, Revue de Turcologie 1936...). Perdure certes une francophonie de la presse, mais celle-ci ne reflète plus une pluralité culturelle et sociale, laquelle est remisée derrière l'injonction de la construction d'une nation unitaire. En pôle de résistance, il faut attendre 1934 pour voir Le Stamboul se mettre au goût du jour et actualiser son titre pour devenir Istanbul.

\section{Bibliographie}

\section{Monographies}

Alemdar Korkmaz, 1975, Istamboul 1875-1964, Histoire d'un journal d'expression française publié en Turquie, Thèse de doctorat, université de Strasbourg.

Deny Jean, Marchand René, 1933, Petit manuel de la Turquie nouvelle, Éd. Jacques Haumont \& cie, Paris.

\section{Articles et contributions à des ouvrages}

BorataV Korkut, 1981, "Kemalist economic policies and étatism" in Kazancigil Ali, Özbudun Ergun, Atatürk, the Founder of a Modern State, Hurst and Cie, London p. 146-165.

Davison Roderic, 1990, “The French Language as a Vehicule for Ottoman reform in the Nineteenth Century" in BACQuéGrammont JeanLouis, Eldem Edhem, De la révolution française à la Turquie d'Atatürk, Éd. Isis, Istanbul, p. 125-141, 286 p.

Deny Jean, « La Presse de Turquie en juillet 1925 », Revue du Monde musulman, t. $61,3^{\circ}$ trimestre 1925 , Paris.

Dumont Paul, 1981, « À l'aube du rapprochement francoturc : le Colonel Mougin, premier représentant de la France auprès du gouvernement d'Ankara [1922-1925] » in La Turquie et à la France à l'époque d'Atatürk, Collection Turcica n ${ }^{\circ}$ 1, p. 58-75. 
Études « Livres et Journaux », Revue Commerciale du Levant, n 201, août 1905, Constantinople.

GEORGEON François, 1985, « La presse de langue française entre les deux guerres mondiales 1919-1939 » in Groc Gérard et ÇAglar Ibrahim, La Presse française de Turquie de 1795 à nos jours, Histoire et Catalogue, Éd. Isis, Istanbul, p. 27-40, 235 p.

Groc Gérard, 1986, « La presse turque et son opinion sur la France, un témoignage du Bulletin de la Presse de l'Ambassade de France [1925-1927] » in Batu Hamit, Bacqué-Grammont Jean-Louis, L'Empire ottoman, la République de Turquie et la France, Éd. Isis, Istanbul, p. 479-498, 700 p.

Groc Gérard, 1992, « Le Journal de Constantinople ou l'ambiguïté du cosmopolitisme, 1843-1853 » in Clayer Nathalie, Popoviç Alexandre, Zarcone Thierry, Presse turque et presse de Turquie, Éd. Isis, Istanbul, p. $15-28,336$ p.

Inal Tanju, KaYA Mümkaz, 2007, «La Turquie Kemaliste, voie/voix francophone dans la Turquie kémaliste 1934-1948 », Colloque université de Galatasaray, nov. 2006, nº 38/39, Documents pour l'Histoire du français langueétrangère.

Kologlu Orhan, 1992, « Le premier journal officiel en français à Istanbul et ses répercussions en Europe » in Clayer Nathalie, Popoviç Alexandre, Zarcone Thierry, Presse turque et presse de Turquie, Éd. Isis, Istanbul, p. 314.

Résumé : les débuts de la République turque, proclamée en octobre 1923, sont, pour la presse en français qui existe en Turquie depuis fort longtemps, un renversement des conditions d'exercice. Ils marquent le passage d'un ancien régime - où elle connaissait depuis plus d'un siècle un succès pérenne et avait acquis ses habitudes - à un nouveau régime. Processus révolutionnaire et transformateur puisqu'il procède d'une guerre d'indépendance où une rébellion nationaliste reconquiert pas à pas (mai 1919-août 1922) un territoire que la volonté des vainqueurs de la Première Guerre mondiale avait considérablement réduit. Un tel recentrage des priorités vers l'affirmation d'une primauté nationale et l'exigence d'une reconnaissance d'indépendance relativise forcément l'utilité d'une presse de langue étrangère, éditée de surcroît dans la langue de l'un des vainqueurs. Pourtant, l'usage perdure et, une fois la paix retrouvée et la république proclamée, 


\section{CAHIERS BALKANIQUES}

30 La presse allophone dans les Balkans

une telle presse continue d'exister dans une grande diversité des styles; mais son utilisation doit se soumettre aux conditions édictées par une volonté politique de plus en plus centralisatrice, au point d'en devenir peu à peu un instrument de propagande.

Mots-clefs : Empire ottoman, presse francophone, Turquie

Abstract: The beginnings of the Turkish Republic, proclaimed in October 1923, are for the French press, which has existed in Turkey for a very long time, as a reversal of the exercising conditions. They actually show the transition from an old regime-where this press has had lasting success for more than a century and had acquired its habits - to a new regime. Revolutionary and transformative process since it proceeds from a war of independence where a nationalist rebellion demanded step by step (May 1919-August 1922) a territory which became the will of the winners of the First World War had considerably reduced. Such a refocusing on priorities towards the affirmation of national supremacy and the requirement for recognition of national independence relativizes the usefulness of a foreign language press including one published in the language of one of the winners. However, the use continues, and once peace has been restored and the republic has been proclaimed, such a foreign press continues to exist within a wide variety of styles; but its use as the point of an instrument of propaganda which has gradually become submitted to the conditions laid down through the will of an increasingly political centralization.

Keywords: French speaking Press, Ottoman Empire, Turkey

Özet: Ekim 1923'de ilanı gerçekleşen Türk Cumburiyeti'nin başlangıçdönemleri, yapılan tüm çabaların tam tersine uzun zamandan beri Türkiye'de varliğın sürdüren Fransizca basın dönemi olmuştur. Bu durum, bir yüzyıldan fazla devam eden bu basinın devam eden başarısın ve onun deneyimlerini/ alışkanlıkların almıs olan eski rejimden yeni rejime geçiş noktasin gösteriyordu. Tüm bu devrimsel ve dönüsümsel süreç, ulusalcı isyanın adım adım 1. Dünya Savaşı'nın galiplerinin arzusu olan topră̆ı talep etmesiyle başlayan (Mayıs 1919-Ă̆ustos 1922) bă̆ımsızlık savaşının devam etmesinden dolayı önemli olarak azaldı. Bă̆ımsızlı̆̆ın tanınma isteği ve ulusal üstünlüğ̈̈n onaylanmasına doğru, bu gibi önceliklere tekrar odaklanılması, savaştan galip çıkanlardan birinin anadili olmasının yan sira yabancı bir dilin basin hayatında kullanılmasın hemen hemen görece duruma getirmiştir. Ancak, bu kullanım devam ederken, barıs săglanı cumburiyet ilan edilir edilmez, aralarındaki geniş çaplı model/yöntem farklilıklarına rağmen bunun gibi yabancı basının varlı̆̆ı da devam eder. Ama, bu kullanım şekli, yavaş yavaş bir propaganda aracı olması noktasina gelerek, gittikçe gücü artmakta olan merkezi

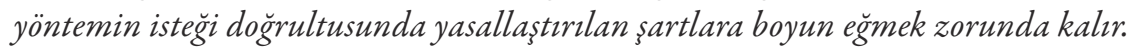


LA PRESSE FRANCOPHONE DANS L'EMPIRE OTTOMAN

ET LA TURQUIE DE MUSTAFA KEMAL $\mathbf{3 1}$

Gérard GROC

Anabtar kelimeler: Fransız basın, Osmanl İmparatorluğu, Türkiye

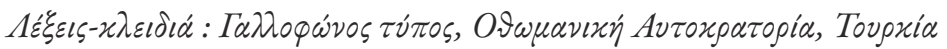

Клучни зборови: Отоманска империја, Франиуски печат, Туриија 